\title{
Can Tests Based on Option Hedging Errors Correctly Identify Volatility Risk Premia?
}

\author{
Nicole Branger ${ }^{\S}$ \\ Christian Schlag§
}

This version: January 15, 2004

\begin{abstract}
This paper provides an in-depth analysis of the properties of popular tests for the existence and the sign of the market price of volatility risk. These tests are frequently based on the fact that for some option pricing models under continuous hedging the sign of the market price of volatility risk coincides with the sign of the mean hedging error. Empirically, however, these tests suffer from both discretization error and model mis-specification. We show that these two problems may cause the test to be either no longer able to detect additional priced risk factors or to be unable to identify the sign of their market prices of risk correctly. Our analysis is performed for the model of Black and Scholes (1973) (BS) and the stochastic volatility (SV) model of Heston (1993). In the model of BS, the expected hedging error for a discrete hedge is positive, leading to the wrong conclusion that the stock is not the only priced risk factor. In the model of Heston, the expected hedging error for a hedge in discrete time is positive when the true market price of volatility risk is zero, leading to the wrong conclusion that the market price of volatility risk is positive.

If we further introduce model mis-specification by using the BS delta in a Heston world we find that the mean hedging error also depends on the slope of the implied volatility curve and on the equity risk premium. Under parameter scenarios which are similar to those reported in many empirical studies the test statistics tend to be biased upwards. The test often does not detect negative volatility risk premia, or it signals a positive risk premium when it is truly zero. The properties of this test furthermore strongly depend on the location of current volatility relative to its long-term mean, and on the degree of moneyness of the option. As a consequence tests reported in the literature may suffer from the problem that in a time-series framework the researcher cannot draw the hedging errors from the same distribution repeatedly. This implies that there is no guarantee that the empirically computed $t$-statistic has the assumed distribution.
\end{abstract}

\section{JEL: G12, G13}

Keywords: Stochastic Volatility, Volatility Risk Premium, Discretization Error, Model Error

\footnotetext{
${ }^{\S}$ Both authors are from the Faculty of Economics and Business Administration, Goethe University, Mertonstr. 17/Uni-Pf 77, D-60054 Frankfurt am Main, Germany. E-mail: branger@wiwi.uni-frankfurt.de, schlag@wiwi.uni-frankfurt.de
} 


\section{Introduction and Motivation}

Explaining excess asset returns is at the core of a discipline financial economists call 'asset pricing'. Naturally, researchers have also become interested in the properties of excess returns on options, see for example the paper by Coval and Shumway (2001). An important question in this context is whether volatility is stochastic, and if so, if volatility risk is priced in the options market. Among others this problem has been investigated by Bakshi and Kapadia (2003) and by Buraschi and Jackwerth (2001). A more general issue in this context is whether there are any additional risk factors besides stock price risk which are priced in the options market. The researcher would like to perform the most general test of this hypothesis possible, and this test should be viable for a whole class of models. Below we will basically consider the whole class of two-dimensional diffusion models with stochastic volatility (SV), containing as special cases the approaches proposed by Heston (1993) or Schöbel and Zhu (1999).

The basis for the empirical analysis of Buraschi and Jackwerth (2001) is the fact that one should be able to span the pricing kernel using only two assets if there are no additional risk factors besides the stock. Their empirical result is that there are other sources of risk implying that deterministic volatility models are mis-specified. Coval and Shumway (2001) find that option returns cannot be explained by the risk-free interest rate and stock returns so that there must be at least one additional risk factor. Finally, Bakshi and Kapadia (2003) show for their sample that options cannot be hedged by the stock and the money market account, again leading to the conclusion that there are other factors besides pure stock price risk. So the general testing methodology is to try to explain the excess option return by the excess stock return (either directly or via a hedge portfolio) and (in case this turns out to be impossible) to conclude that there are other risk factors at least one of which is priced.

It is important to note that it is not a priori clear what sign the market price of volatility risk should have in equilibrium. Whereas risk-averse investors would certainly demand a premium for bearing stock price risk, this is not so clear in the case of volatility 
risk. Investors might be averse to volatility risk, since it will in general introduce additional variation into their consumption stream, which would lead to a positive risk premium. On the other hand, one could imagine a situation where after a large negative shock to aggregate wealth, investors would be willing to pay a premium for assets with a high exposure to volatility risk, because these would offer the chance to reach higher levels of consumption sooner than assets with lower volatility. According to this argument we would expect a negative risk premium for volatility. As a consequence we do not have a clear null hypothesis for the sign of the market price of volatility risk, and its determination becomes a purely empirical matter. In the literature, there are by now standard empirical test procedures designed to identify the sign of the volatility risk premium. Our paper is not empirical in the strict sense of the word, since we do not perform an empirical study of our own. Instead we analyze the validity of these standard tests.

As shown in Bakshi and Kapadia (2003) and as will be discussed below in detail the sign of the volatility risk premium is (under certain ideal conditions) the same as the sign of the mean hedging error. Bakshi and Kapadia (2003) build on this result and construct a hedge portfolio for a European call option by going long the number of stocks given by the BS delta and by borrowing the necessary remaining funds at the risk-free rate. They then test the null hypothesis that the hedging error generated by this strategy has zero mean and conclude in the case of rejection that there is a non-zero volatility risk premium with a sign identical to that of the mean hedging error. The use of the BS hedging coefficient is justified by the authors in this context as a choice which is robust against mis-specification of an SV model. Note that, as opposed to standard risk management approaches, the goal of this approach is not to find a minimum variance hedge for an option, but solely to use the sign of the mean hedging error as an identification criterion for the sign of the volatility risk premium.

In general the described test is correct, i.e. the signs of the mean hedging error and the volatility risk premium coincide, under ideal conditions, when trading is actually continuous and when the correct model is used to calculate the 'delta', i.e. the partial derivative of the option price with respect to the stock price. In empirical studies both 
assumptions are usually violated. The hedging strategy is not implemented in continuous time, but trading is only possible at discrete points in time. This leads to a discretization error. Furthermore the true data generating process is unknown, which in general creates a model error, since it is no longer possible to compute the true hedge portfolio. For example, the delta of the option might be computed under the assumptions of the Black and Scholes (1973) model (BS herafter), whereas in reality the dynamics of the economy are given by the Heston (1993) SV model. In addition, the expected hedging error (EHE) is usually estimated as a time-series average in empirical studies. This procedure is based on the implicit assumption that each hedging error is drawn out of the same distribution, which does not hold in reality due to the dependence of the hedging error on current volatility and on the moneyness of the option, as we will show below.

After deriving a number of theoretical results concerning the EHE for a call option in a variety of scenarios we perform a simulation study to get a better idea of the properties of option hedging errors under discretization error and model mis-specification. The data generating process is given by the Heston (1993) model which is one of the most popular approaches to SV option pricing. If the hedging test is supposed to work for a large class of SV models it should be able to detect the sign of the volatility risk premium also in the special case of the Heston model. So our simulation study is at the same time an (indirect) check of the claim made by Bakshi and Kapadia (2003), namely that their test procedure is rather robust to the (mis-)specification of the SV process.

In the case of continuous trading and when BS is the true model the use of the BS delta creates a perfect hedge, i.e. the realized hedging error is identically equal to zero. When we are faced with trading restrictions such that the portfolio can only be rebalanced at discrete points in time the market becomes incomplete and a perfect hedge is no longer possible, since there is a discretization error. The use of the hedge ratio derived in the continuous case then results in a systematic bias. For a convex claim, the EHE can be shown to be always positive. It can be forced to equal zero, however, when a certain special hedge ratio different from the standard delta is applied. We will explicitly derive this hedge ratio which we call the mean zero hedge ratio. 
For the Heston model we first derive a number of analytical results concerning the EHE. Here we find that the sign of the EHE is equal to the sign of the volatility risk premium only in an economy with continuous trading and if the correct delta is used. This property does not carry over to the situation with discrete trading. Again there is a discretization error, but in contrast to the BS model the sign of this error is no longer unique and can only be determined for some special cases. For a zero market price of volatility risk and discrete hedging the EHE is positive, if the partial derivative from the Heston model is used as the hedge ratio. As in the BS model the EHE can be forced to equal zero if the hedge ratio is adjusted appropriately. However, this is no longer true if the market price of volatility risk is not zero. There is no longer a systematic relationship between the sign of the EHE and the sign of the market price of volatility risk, and there is also no closed-form adjustment to the hedge ratio as opposed to the BS case.

When there is also model error, i.e. when the BS delta is used in an SV economy, the sign of the EHE does not necessarily equal the sign of the market price of volatility risk anymore, even when the hedge portfolio is rebalanced continuously. The additional bias due to model mis-specification depends on the equity risk premium and on the slope of the implied volatility curve. Given the empirical evidence on stock and option markets around the world the most important scenario here is certainly given by a downward sloping smile curve and a positive equity risk premium. In this setting model error tends to increase the EHE so that the test too often falsely concludes that the volatility risk premium is positive when it is actually zero or negative.

The remainder of the paper is organized as follows. In section 2 the main idea of hedging based tests is described. Section 3 provides an analytical comparison of different hedge strategies in the BS model together with some numerical results on hedging errors. In section 4 we perform an analogous investigation for the Heston model and assess the impact of model error on option hedging errors. Section 5 concludes. 


\section{An Overview of Tests for Volatility Risk Premia}

\subsection{The Relationship between Hedging Error and Risk Pre- mium}

As stated in the introduction one of the most important issues in derivative pricing is whether volatility is stochastic and, if so, whether the market price of volatility risk is different from zero.

The main idea of the test for the existence of a volatility risk premium is that the expected return on an option can be decomposed into the risk-free rate of interest plus the sum of the risk premia for stock price risk, volatility risk, and other types of risk. So if the expected excess option return can be perfectly explained by the expected excess stock return, two scenarios are possible. Either there are no other risk factors besides stock price risk (as it is the case in the BS model), or there are other risk factors, but they are not priced, i.e. investors do not require an additional compensation for bearing these types of risk. The converse is that if expected excess option returns cannot be explained by expected excess stock returns, there are additional risk factors, at least one of which is priced.

This line of argument leads to two testable implications. First one can investigate whether excess option returns can be explained by excess returns of the underlying. This yields the test equation

$$
\frac{C_{t+\tau}}{C_{t}}-e^{r \tau}=\beta_{t}\left(\frac{S_{t+\tau}}{S_{t}}-e^{r \tau}\right)+\epsilon_{t+\tau}
$$

with $C_{t}$ and $S_{t}$ representing the price of the option and the stock at time $t$, respectively. $\epsilon_{t+\tau}$ is the error term, and $\beta_{t}$ denotes some measure of risk. The test focuses on the properties of the error term $\epsilon_{t+\tau}$. It is identically equal to zero if the option return can be perfectly explained by the stock return and if the appropriate $\beta_{t}$ is chosen. It will have realizations different from zero if there are additional risk factors like stochastic volatility or if an inappropriate coefficient $\beta_{t}$ is chosen. It will also be different from zero if, in a continuous time model, we consider the realized returns over discrete intervals. 
The really important issue, however, is whether the mean of $\epsilon_{t+\tau}$ is equal to zero or not. The mean of the error term is just the part of the expected excess option return that is not explained by the expected excess stock return. As argued above, if this expectation is different from zero, there are other risk factors besides stock price risk, and at least one of these factors is priced. The test therefore amounts to using the mean error to make inferences about the market prices of risk for additional risk factors.

As an alternative to the approach described by equation (1), one can set up a hedge portfolio for the option containing the stock and the money market account. Using an argument analogous to the one presented above this leads to the test if the error term $\widehat{\epsilon}_{t+\tau}:=C_{t} \epsilon_{t+\tau}$ in the equation

$$
C_{t+\tau}=C_{t} e^{r \tau}+\beta_{t} \frac{C_{t}}{S_{t}}\left(S_{t+\tau}-e^{r \tau} S_{t}\right)+\widehat{\epsilon}_{t+\tau}
$$

has zero mean. Again, we conclude that if the expected hedging error is zero only stock price risk carries a premium, whereas in the opposite case there must be some other priced risk factor. As one can see, equations (1) and (2) are just multiples of each other. The researcher can thus choose which specification should be used for the test, and for the rest of the paper we will focus on hedging errors of the type shown in equation (2).

The test procedures described above ignore at least two problems. First, by applying in discrete time hedge ratios or risk measures which were derived in a continuous-time context a discretization error is introduced. If this discretization error has a systematic impact on the properties of the mean hedging error, the test may become invalid. This problem raises the additional question whether there is at all a discrete hedge ratio that can ensure a zero mean error. Second, since the true data generating process is unknown, the test may also suffer from model mis-specification. The hedge ratio depends on the option pricing model, and if the wrong model is used, the hedge ratio will in general be no longer correct. In particular, the use of the BS delta may induce a bias when volatility is stochastic.

As will be shown below the distribution of the hedging error depends on the moneyness and maturity of the option as well as on the current values of the state variables. This 
creates another problem in empirical applications of the above testing principle. When we calculate the mean hedging error from a time series the individual errors will usually be drawn from different distributions. It is thus unclear whether the mean hedging error from a time series can actually tell the researcher anything at all about the market prices of risk. We will avoid these problems in our simulation study and instead focus on the discretization error and the impact of model mis-specification.

\subsection{Hedging Strategy and Hedging Error}

A hedging strategy for a contingent claim consists of investments in the two traded basis assets, the stock and the money market account. The number of shares held at time $t$ is denoted by $H_{t}$, and the investment in the money market account simply represents the residual and serves to make the strategy self-financing. The value $\Pi_{t}$ of the hedge portfolio at time $t$ is given by the following expression:

$$
\Pi_{t}=e^{r t}\left(\Pi_{0}+\int_{0}^{t} e^{-r u} H_{u}\left(d S_{u}-r S_{u} d u\right)\right)
$$

where $\Pi_{0}$ is the initial value.

The hedging error is defined as the difference between the claim price and $\Pi_{t}$, and one obtains

$$
C_{t}-\Pi_{t}=e^{r t}\left(C_{0}-\Pi_{0}+\int_{0}^{t} e^{-r u}\left(d C_{u}-r C_{u} d u\right)-\int_{0}^{t} e^{-r u} H_{u}\left(d S_{u}-r S_{u} d u\right)\right)
$$

so that the hedging error is positive when the value of the claim is greater than the value of the hedge portfolio. In a complete market we can, for any contingent claim, choose a hedging strategy that has a value process equal to the value process of the claim. The resulting hedging error would then by identically equal to zero.

The quantity

$$
\begin{aligned}
D(t, t+\tau) & =\left(C_{t+\tau}-\Pi_{t+\tau}\right)-e^{r \tau}\left(C_{t}-\Pi_{t}\right) \\
& =e^{r(t+\tau)}\left(\int_{t}^{t+\tau} e^{-r u}\left(d C_{u}-r C_{u} d u\right)-\int_{t}^{t+\tau} e^{-r u} H_{u}\left(d S_{u}-r S_{u} d u\right)\right)
\end{aligned}
$$


represents the hedging error over the interval $[t, t+\tau]$, computed at time $t+\tau$. In principle one could compute the total hedging error from time $t=0$ to some point in time $t_{i}$, where $0=t_{0}<t_{1}<\ldots<t_{i}$, according to the following equation:

$$
C_{t_{i}}-\Pi_{t_{i}}=\left(C_{0}-\Pi_{0}\right) e^{r t_{i}}+D\left(t_{0}, t_{1}\right) e^{r\left(t_{i}-t_{1}\right)}+D\left(t_{1}, t_{2}\right) e^{r\left(t_{i}-t_{2}\right)}+\ldots+D\left(t_{i-1}, t_{i}\right) .
$$

In the analysis below, however, we will exclusively focus on one-period hedging errors and their statistical properties. In terms of notation, the hedging error over some finite interval $[t, t+\tau]$ is denoted by $D^{c}(t, t+\tau)$ for continuous trading and by $D^{d}(t, t+\tau)$ for discrete trading. When trading is discrete, the hedge ratio has to be held constant over every subperiod, i.e. the hedge ratio from time $t_{i}$ to time $t_{i+1}$ is held fixed at some value $H_{t_{i}}$. This implies in particular that the discrete hedging error is given by

$$
D^{d}\left(t_{i}, t_{i+1}\right)=C_{t_{i+1}}-C_{t_{i}} e^{r\left(t_{i+1}-t_{i}\right)}-H_{t_{i}}\left(S_{t_{i+1}}-S_{t_{i}} e^{r\left(t_{i+1}-t_{i}\right)}\right)
$$

\section{$3 \quad$ Hedging Error in the Model of Black and Scholes}

In the BS model the dynamics of the two basis assets $S$ (the stock) and $B$ (the money market account) are given by

$$
\begin{aligned}
& d S_{t}=\mu S_{t} d t+\sigma S_{t} d W_{t} \\
& d B_{t}=r B_{t} d t, \quad B_{0} \equiv 1
\end{aligned}
$$

where $d W_{t}$ represents the increments of a standard Brownian motion. The associated dynamics under the risk-neutral measure are given by

$$
d S_{t}=r S_{t} d t+\sigma S_{t} d \widetilde{W}_{t}
$$

with $d \widetilde{W}_{t}$ again as a standard Brownian motion. Since the dynamics of $B$ do not contain a stochastic term they are not affected by the change of measure.

The test described in section 2 is based on the EHE. We will now analyze the EHE in the BS model for different hedging strategies and for both continuous and discrete 
trading. We start with the formula for the EHE for a general European claim and a general hedge ratio $H$, before we focus on call options and set the hedge ratio equal to the partial derivative of the claim price with respect to the stock price and additionally for discrete trading - to the so-called mean-zero hedge ratio.

The EHE for the BS model is given in the following proposition.

Proposition 1 (BS: EHE for continuous trading) The true model is the model of Black Scholes. The price of the claim is denoted by $C_{t}^{B S}=c^{B S}\left(t, S_{t}\right)$, where $c^{B S}\left(t, S_{t}\right)$ is short for the function $c^{B S}(u, s)$ evaluated at $u=t$ and $s=S_{t}$. Trading takes place continuously, the hedge ratio at time $t$ is $H_{t}$. Then, the EHE over the interval $[t, t+\tau]$ is given by

$$
\begin{aligned}
& E^{P}\left[D^{c}(t, t+\tau) \mid \mathcal{F}_{t}\right]= \\
& \quad(\mu-r) \int_{t}^{t+\tau} e^{r(t+\tau-u)} E^{P}\left[\left(\frac{\partial c^{B S}}{\partial s}\left(u, S_{u}\right)-H_{u}\right) S_{u} \mid \mathcal{F}_{t}\right] d u
\end{aligned}
$$

with $E^{P}$ denoting the expectation under the physical measure and $\mathcal{F}_{t}$ representing information available at time $t$.

Proof: The price of the European claim $c^{B S}\left(t, S_{t}\right)$ has to satisfy the partial differential equation

$$
\frac{\partial c^{B S}}{\partial t}+\frac{\partial c^{B S}}{\partial s} r s+\frac{1}{2} \frac{\partial^{2} c^{B S}}{\partial s^{2}} \sigma^{2} s^{2}=r c^{B S}
$$

Applying Ito's lemma to the price of a contingent claim yields the dynamics of $C^{B S}$,

$$
\begin{aligned}
d C_{t}^{B S} & =\frac{\partial c^{B S}}{\partial t} d t+\frac{\partial c^{B S}}{\partial s} d S_{t}+\frac{1}{2} \frac{\partial^{2} c^{B S}}{\partial s^{2}} \sigma^{2} S_{t}^{2} d t \\
& =r C_{t}^{B S} d t+\frac{\partial c^{B S}}{\partial s}\left(d S_{t}-r S_{t} d t\right)
\end{aligned}
$$

where the second equality follows from (6). Plugging equation (7) for the change in the price of the derivative into the general formula (3) for the realized hedging error one obtains

$$
D^{c}(t, t+\tau)=\int_{t}^{t+\tau} e^{r(t+\tau-u)}\left(\frac{\partial c^{B S}}{\partial s}\left(u, S_{u}\right)-H_{u}\right)\left(d S_{u}-r S_{u} d u\right)
$$


After taking expectations the proposition follows.

Note that the previous proposition refers to an arbitrary hedge ratio $H_{t}$. Setting the hedge ratio equal to the partial derivative, i.e. choosing

$$
H_{t} \equiv \frac{\partial c^{B S}}{\partial s}\left(t, S_{t}\right)
$$

yields a hedging error which is identically equal to zero, as we can see directly from equation (8). Of course, this result is not surprising. In the BS model there is a perfect hedge with zero error for every claim satisfying certain regularity conditions, with an optimal hedge ratio equal to the partial derivative of the claim price with respect to the stock price.

\subsection{Discretization Error}

If we now introduce the restriction that trading is only possible at discrete points in time $t_{i}$, the properties of the expected hedging error change. We obtain

Proposition 2 (BS: EHE for discrete trading) The true model is BS. Trading takes place at the discrete dates $0=t_{0}, t_{1}, t_{2}, \ldots, t_{n}=T$, the hedge ratio at time $t_{i}$ is $H_{t_{i}}$. Then the EHE over the interval $\left[t_{i}, t_{i+1}\right]$ is

$$
\begin{aligned}
E^{P}[ & \left.D^{d}\left(t_{i}, t_{i+1}\right) \mid \mathcal{F}_{t_{i}}\right] \\
= & (\mu-r)\left(\frac{\partial c^{B S}}{\partial s}\left(t_{i}, S_{t_{i}}\right)-H_{t_{i}}\right) \int_{t_{i}}^{t_{i+1}} e^{r\left(t_{i+1}-t\right)} E^{P}\left[S_{t} \mid \mathcal{F}_{t_{i}}\right] d t \\
& \quad+(\mu-r)^{2} \int_{t_{i}}^{t_{i+1}} e^{r\left(t_{i+1}-t\right)} E^{P}\left[S_{t} \mid \mathcal{F}_{t_{i}}\right] E^{P^{S}}\left[\int_{t_{i}}^{t} \frac{\partial^{2} c^{B S}}{\partial s^{2}}\left(u, S_{u}\right) S_{u} d u \mid \mathcal{F}_{t_{i}}\right] d t .
\end{aligned}
$$

Here $E^{P^{S}}$ denotes the expectation under the measure $P^{S}$ defined via the Radon-Nikodym derivative

$$
\left.\frac{d P^{S}}{d P}\right|_{\mathcal{F}_{t}}=\frac{S_{t}}{E^{P}\left[S_{t}\right]} .
$$

Proof: See the appendix. 
As shown above applying a hedge ratio equal to the partial derivative of the claim price with respect to the stock price generates a zero hedging error in the case of continuous trading. This is no longer the case with discrete trading, since technically speaking the market becomes incomplete. Using the partial derivative as the hedge ratio, i.e. setting

$$
H_{t_{i}} \equiv \frac{\partial c^{B S}}{\partial s}\left(t_{i}, S_{t_{i}}\right)
$$

we obtain the following corollary.

Corollary 1 (BS: Discretization error) The true model is BS. Trading takes place at the discrete dates $0=t_{0}, t_{1}, t_{2}, \ldots, t_{n}=T$, the hedge ratio is the partial derivative. Then the EHE over the interval $\left[t_{i}, t_{i+1}\right]$ is given by

$$
\begin{aligned}
& E^{P}\left[D^{d}\left(t_{i}, t_{i+1}\right) \mid \mathcal{F}_{t_{i}}\right] \\
& =(\mu-r)^{2} \int_{t_{i}}^{t_{i+1}} e^{r\left(t_{i+1}-t\right)} E^{P}\left[S_{t} \mid \mathcal{F}_{t_{i}}\right] E^{P^{S}}\left[\int_{t_{i}}^{t} \frac{\partial^{2} c^{B S}}{\partial s^{2}}\left(u, S_{u}\right) S_{u} d u \mid \mathcal{F}_{t_{i}}\right] d t .
\end{aligned}
$$

It is obvious that if the price of the claim is a convex function of the stock price, the discretization error will be positive for any non-vanishing equity risk premium. As shown by Bergman, Grundy, and Wiener (1996) the price of a claim is a convex function of the stock price for any path-independent claim with a convex payoff function. For a European call option, the EHE is therefore positive (see also Dudenhausen (2002)). Note that our result differs from Boyle and Emanuel (1980) who use limiting arguments improperly to arrive at the conclusion that the discretization error vanishes even in discrete time.

The result in Corollary 1 has important consequences for the test described in section 2. This test is based on the idea that the EHE is zero if and only if equity risk is the only priced risk factor. The (wrong) conclusion derived from such a hedging based test in the BS model must be that equity risk is not the only priced factor, but that there is some other source of risk with a positive premium (given that the number of observations is large enough to make the mean significantly positive in an empirical application).

Of course, the absolute amount of the EHE depends on the length of the time interval between two consecutive trading dates (see also Bertsimas, Kogan, and Lo (2000)). It 
becomes smaller the shorter this period, but irrespective of the length of the trading interval it always remains analytically strictly positive for non-zero equity risk premia.

\subsection{Correcting for the Discretization Error}

Given the results in the previous subsection the question now is whether there is any hedge ratio that would generate a zero mean hedging error even with discrete trading. The next proposition shows that we can indeed determine such a hedge ratio.

Proposition 3 (BS: Mean-zero hedge ratio) The true model is BS. Trading takes place at the discrete dates $0=t_{0}, t_{1}, t_{2}, \ldots, t_{n}=T$. The mean-zero hedge ratio is defined as

$$
\begin{aligned}
& H_{t_{i}}^{B S, M Z}\left(t_{i+1}\right) \\
& \quad=\frac{\partial c^{B S}}{\partial s}\left(t_{i}, S_{t_{i}}\right)+\frac{\int_{t_{i}}^{t_{i+1}} e^{-r t} E^{P}\left[S_{t} \mid \mathcal{F}_{t_{i}}\right] E^{P^{S}}\left[\int_{t_{i}}^{t} \frac{\partial^{2} c^{B S}}{\partial s^{2}}\left(u, S_{u}\right) S_{u} d u \mid \mathcal{F}_{t_{i}}\right] d t}{\int_{t_{i}}^{t_{i+1}} e^{-r t} E^{P}\left[S_{t} \mid \mathcal{F}_{t_{i}}\right] d t}(\mu-r) .
\end{aligned}
$$

Proof: Setting the EHE from Proposition 2 equal to zero and rearranging the equation yields the desired hedge ratio.

The new hedge ratio differs from the partial derivative by the second term. For a convex claim, the fraction is positive so that the mean-zero hedge ratio is greater than the partial derivative for a positive equity risk premium.

This result is intuitively clear. When the hedge ratio is given by the partial derivative, we know that the EHE is positive. Given our definition of the hedging error the expected terminal value of the hedge portfolio is therefore too small. To get an EHE equal to zero, we have to correct the number of stocks such that the expected terminal value of the hedge portfolio increases. For a positive (negative) risk premium, this amounts to increasing (decreasing) the number of stocks. If the claim to be hedged is a European call or put, we can also give a closed form solution for the EHE and for the mean-zero hedge ratio which is based on a formula for the expected price of a call option (see Rubinstein $(1984))$. 
The most important property of the hedge ratio given in Proposition 3 is that based on $H_{t_{i}}^{B S, M Z}\left(t_{i+1}\right)$ the standard test works properly. We would draw the correct conclusion that there is no other priced factor besides the stock. However, this comes at the price of the need to specify the dynamics of the underlying asset both under the risk-neutral and the physical measure, as opposed to the partial derivative which does not depend on $\mu$. In particular, the hedge ratio now explicitly depends on the market price of equity risk.

\section{Stochastic Volatility Model}

The stochastic volatility setup is given by the Heston (1993) model, i.e. under the physical measure the stock price and the local variance are governed by the stochastic differential equations

$$
\begin{aligned}
& d S_{t}=\mu S_{t} d t+\sqrt{V_{t}} S_{t} d W_{t}^{S} \\
& d V_{t}=\kappa\left(\theta-V_{t}\right) d t+\sigma_{V} \sqrt{V_{t}}\left(\rho d W_{t}^{S}+\sqrt{1-\rho^{2}} d W_{t}^{V}\right)
\end{aligned}
$$

where $d W_{t}^{S}$ and $d W_{t}^{V}$ are the increments of two independent Brownian motions, and $\rho$ represents the correlation coefficient between stock returns and volatility changes. The market price of volatility risk is assumed to equal $\lambda_{V} \sqrt{V_{t}}$ so that we obtain the following processes under the risk-neutral measure:

$$
\begin{aligned}
& d S_{t}=r S_{t} d t+\sqrt{V_{t}} S_{t} d \widetilde{W}_{t}^{S} \\
& d V_{t}=\left(\kappa\left(\theta-V_{t}\right)-\sigma_{V} \lambda_{V} V_{t}\right) d t+\sigma_{V} \sqrt{V_{t}}\left(\rho d \widetilde{W}_{t}^{S}+\sqrt{1-\rho^{2}} d \widetilde{W_{t}^{V}}\right) .
\end{aligned}
$$

In what follows we will consider different hedging strategies for a European call option, but for any strategy only the stock and the money market account can be used to form the hedge portfolio. The strategies differ with respect to whether they are adjusted continuously or only at discrete points in time, and they also differ with respect to the hedge ratio which is given by the partial derivative of the call price either from the Heston model or from the (incorrectly assumed) BS model. The characteristics of the EHE will generally depend on both these factors, i.e. both on the discretization error and on potential model mis-specification. 
The EHE is used in the test described in section 2 to learn something about the pricing of risk factors besides stock price risk. The test is based on the hypothesis that the EHE equals zero if the only priced risk factor is the stock, and that it will be different from zero otherwise. Furthermore, if there is only one additional risk factor like it is the case in the Heston model, then the sign of the EHE for a call option is assumed to coincide with the sign of the market price of risk. This is exactly the idea employed by Bakshi and Kapadia (2003) who test whether volatility risk is priced and whether the volatility risk premium is positive or negative.

Both discretization error and model error make the design of this test for volatility risk premia seem questionable. If for some hedging strategy the relationship between the sign of the EHE and the sign of the market prices of risk is not as assumed the test will lead to wrong conclusions. It may either not detect a positive or negative market price of volatility risk, or it may wrongly conclude that volatility risk is priced although it is not.

\subsection{Expected Hedging Error for Continuous Trading}

\subsubsection{Correct model}

We start our analysis for the ideal case of continuous trading. There will be a non-zero hedging error even in this scenario, since the market is incomplete so that a perfect hedge for contracts with non-zero sensitivity to volatility is impossible. We first derive a formula for the EHE for a general claim and for a general hedge ratio which is then studied in detail for the special case where the hedge ratio is equal to the partial derivative from the Heston model. The following proposition gives an expression for the EHE when an arbitrary hedge ratio is employed.

Proposition 4 (Heston: EHE for continuous trading) The true model is Heston. The price of the claim is denoted by $C_{t}^{S V}=c^{S V}\left(t, S_{t}, V_{t}\right)$, where $c^{S V}\left(t, S_{t}, V_{t}\right)$ is short for the function $c^{S V}(u, s, v)$ evaluated at $u=t, s=S_{t}$, and $v=V_{t}$. The hedge portfolio is rebalanced continuously, the hedge ratio at time $t$ is denoted by $H_{t}$. Then the EHE over 
the interval $[t, t+\tau]$ is

$$
\begin{aligned}
E^{P}\left[D^{c}(t, t+\tau) \mid \mathcal{F}_{t}\right]= & (\mu-r) \int_{t}^{t+\tau} e^{r(t+\tau-u)} E^{P}\left[\left(\frac{\partial c^{S V}}{\partial s}\left(u, S_{u}, V_{u}\right)-H_{u}\right) S_{u} \mid \mathcal{F}_{t}\right] d u \\
& +\lambda_{V} \int_{t}^{t+\tau} e^{r(t+\tau-u)} E^{P}\left[\frac{\partial c^{S V}}{\partial v}\left(u, S_{u}, V_{u}\right) \sigma_{V} V_{u} \mid \mathcal{F}_{t}\right] d u
\end{aligned}
$$

Proof: See the appendix.

Setting the hedge ratio equal to the partial derivative of the call price with respect to the stock price, i.e. choosing

$$
H_{t} \equiv \frac{\partial c^{S V}}{\partial s}\left(t, S_{t}, V_{t}\right)
$$

and applying Proposition 4 we obtain

Corollary 2 (Heston: EHE under ideal conditions) The true model is Heston. The hedge portfolio is rebalanced continuously, the hedge ratio is the partial derivative. Then the EHE over the interval $[t, t+\tau]$ is

$$
E^{P}\left[D^{c}(t, t+\tau) \mid \mathcal{F}_{t}\right]=\lambda_{V} \int_{t}^{t+\tau} e^{r(t+\tau-u)} E^{P}\left[\frac{\partial c^{S V}}{\partial v}\left(u, S_{u}, V_{u}\right) \sigma_{V} V_{u} \mid \mathcal{F}_{t}\right] d u
$$

For a European call $\frac{\partial c^{S V}}{\partial v}>0$, and the sign of the EHE is equal to the sign of the market price of volatility risk. Therefore the test described in section 2 is valid under the ideal conditions of continuous trading and the absence of model mis-specification.

The sign of the EHE of a continuous hedge based on the correct model can in general be used to identify the sign of $\lambda_{V}$ if the vega of the claim, i.e. the partial derivative with respect to the current level of the state variable (variance or volatility), has a unique sign, and if the state variable itself remains non-negative with probability one. This second condition can become critical for example in the SV model of Schöbel and Zhu (1999) where the state variable is local volatility (as opposed to the local variance in the Heston model), governed by an Ornstein-Uhlenbeck process.

Note further that our result in Corollary 2 is more general than the corresponding result derived by Bakshi and Kapadia (2003) in their Proposition 2. Whereas they assume 
that the vega of the claim under consideration is proportional to the stock price and independent of local volatility, no such restriction has to be imposed for the derivation of the EHE above. This is a significant advantage, since we are only interested in whether the sign of the volatility premium is the same as the sign of the EHE and not in the exact numerical value of this premium. The properties of the signs of these two quantities, however, can be perfectly derived from the corollary.

\subsubsection{Model Error}

The terms model error and model mis-specification refer to the use of an incorrect hedge model, due to the fact that the true data generating process is unknown. In this paper all hedges under model error are based on the BS model. This seems a natural choice, since this model represents the simplest approach to option pricing, and it has proved to be rather robust in empirical applications (see, e.g., Bakshi, Cao, and Chen (1997)). So the hedge ratio applied in the test for volatility risk premia is given by

$$
H_{t} \equiv \frac{\partial c^{B S}}{\partial s}\left(t, S_{t}\right)
$$

To compute this partial derivative a volatility input is needed. We use the implied BS volatility of the option for this purpose, although other studies use historical measures. Nevertheless, the implied volatility of an option seems preferable to us as the more natural choice given the way practitioners usually handle liquid options.

An expression for the EHE in the Heston model is given in the following proposition.

Proposition 5 (Heston: EHE under model error) The true model is Heston. The hedge portfolio is rebalanced continuously, the hedge ratio is the BS delta based on the implied volatility. $\sigma^{B S}\left(t, M_{t}\right)$ denotes the BS implied volatlity at time $t$ for an option with

moneyness $M_{t}$, where $M_{t}=\frac{K}{S_{t}}$ and $K$ is the strike price. Then the EHE over the interval 
$[t, t+\tau]$ is

$$
\begin{aligned}
E^{P}\left[D^{c}(t, t+\tau) \mid \mathcal{F}_{t}\right] \\
=\lambda_{V} \int_{t}^{t+\tau} e^{r(t+\tau-u)} E^{P}\left[\frac{\partial c^{S V}}{\partial v}\left(u, S_{u}, V_{u}\right) \sigma_{V} V_{u} \mid \mathcal{F}_{t}\right] d u \\
\quad-(\mu-r) \int_{t}^{t+\tau} e^{r(t+\tau-u)} E^{P}\left[\frac{\partial c^{B S}}{\partial \sigma^{B S}}\left(u, S_{u}\right) \frac{\partial \sigma^{B S}}{\partial m}\left(u, M_{u}\right) M_{u} \mid \mathcal{F}_{t}\right] d u .
\end{aligned}
$$

Proof: See the appendix.

The first term in equation (13) is the EHE if the correct hedge ratio is used in continuous time (see Corollary 2). The second term originates from the use of the BS delta instead of the correct partial derivative from the Heston model. It therefore represents the impact of model mis-specification. As can be seen from Proposition 4, the sign of this term depends on the expected difference between the partial derivative in the Heston model and the partial derivative in the BS model as well as on the risk premium of the stock. Equation (22) in the appendix shows that the difference between the partial derivatives is related to the slope of the implied volatility function via

$$
\frac{\partial c^{S V}}{\partial s}\left(t, S_{t}, V_{t}\right)-\frac{\partial c^{B S}}{\partial s}\left(t, S_{t}\right)=-\frac{\partial c^{B S}}{\partial \sigma}\left(t, S_{t}\right) \cdot \frac{\partial \sigma^{B S}}{\partial m}\left(t, M_{t}\right) \cdot \frac{M_{t}}{S_{t}} .
$$

Empirically, the risk premium on stocks is generally found to be positive, and the smile for most stocks and indices decreases in the strike price of the options. These stylized facts imply that the partial derivative in the Heston model is greater than the partial derivative in the BS model. Both Proposition 4, which is based on the expected differences between the partial derivatives, and equation (13), which is based on the slope of the smile, show that under these conditions the expected hedging error increases compared to the expected hedging error when the correct Heston delta is used.

If the market price of volatility risk is zero the EHE in the above proposition reduces to the second term which can be attributed entirely to model error. If the market price of stock risk is positive, and if the implied volatility is a decreasing function of the strike price, the EHE due to model risk will be positive. A test for the sign of the volatility risk premium based on the EHE will therefore tend to reject the null hypothesis of a zero risk premium against the alternative of a positive $\lambda_{V}$. 
In scenarios with a non-zero volatility premium the sign of the EHE will no longer coincide with the sign of the market price of volatility risk, at least not in general. The test from section 2 is therefore not able to identify the sign of the market price of volatility risk properly.

\subsection{Expected Hedging Error for Discrete Trading}

\subsubsection{Pure Discretization Error}

We now analyze the EHE when the hedge is rebalanced only at discrete points in time. First, we derive a general formula for the EHE before we take a closer look at the hedging error for particular choices of the hedge ratio.

\section{Proposition 6 (Heston: EHE under discretization error for arbitrary hedge ratio)}

The true model is Heston. Trading takes place at the discrete dates $0=t_{0}, t_{1}, t_{2}, \ldots, t_{n}=$ $T$, the hedge ratio at time $t_{i}$ is $H_{t_{i}}$. Then the EHE over the interval $\left[t_{i}, t_{i+1}\right]$ is

$$
\begin{aligned}
E^{P}\left[D^{d}\left(t_{i}, t_{i+1}\right) \mid \mathcal{F}_{t_{i}}\right] & \lambda_{V} \int_{t_{i}}^{t_{i+1}} e^{r\left(t_{i+1}-t\right)} E^{P}\left[\frac{\partial c^{S V}}{\partial v}\left(t, S_{t}, V_{t}\right) \sigma_{V} V_{t} \mid \mathcal{F}_{t_{i}}\right] d t \\
& +(\mu-r)\left(\frac{\partial c^{S V}}{\partial s}\left(t_{i}, S_{t_{i}}, V_{t_{i}}\right)-H_{t_{i}}\right) \int_{t_{i}}^{t_{i+1}} e^{r\left(t_{i+1}-t\right)} E^{P}\left[S_{t} \mid \mathcal{F}_{t_{i}}\right] d t \\
& +(\mu-r)^{2} \int_{t_{i}}^{t_{i+1}} e^{r\left(t_{i+1}-t\right)} E^{P}\left[S_{t} \mid \mathcal{F}_{t_{i}}\right] E^{P^{S}}\left[\int_{t_{i}}^{t} \frac{\partial^{2} c^{S V}}{\partial s^{2}}\left(u, S_{u}, V_{u}\right) S_{u} d u \mid \mathcal{F}_{t_{i}}\right] d t \\
& +(\mu-r) \lambda_{V} \int_{t_{i}}^{t_{i+1}} e^{r\left(t_{i+1}-t\right)} E^{P}\left[S_{t} \mid \mathcal{F}_{t_{i}}\right] E^{P^{S}}\left[\int_{t_{i}}^{t} \frac{\partial^{2} c^{S V}}{\partial s \partial v}\left(u, S_{u}, V_{u}\right) \sigma_{V} V_{u} d u \mid \mathcal{F}_{t_{i}}\right] d t
\end{aligned}
$$

Proof: See the appendix.

Setting the hedge ratio equal to the partial derivative of the claim price with respect to the stock price computed in the Heston framework, i.e. setting

$$
H_{t_{i}} \equiv \frac{\partial c^{S V}}{\partial s}\left(t_{i}, S_{t_{i}}, V_{t_{i}}\right)
$$

we obtain 
Corollary 3 (Heston: Discretization error for delta hedge) The true model is Heston. Trading takes place at the discrete dates $0=t_{0}, t_{1}, t_{2}, \ldots, t_{n}=T$, the hedge ratio is the Heston partial derivative of the claim price with respect to the stock price. Then the EHE over the interval $\left[t_{i}, t_{i+1}\right]$ is given by

$$
\begin{aligned}
E^{P}[ & \left.D^{d}\left(t_{i}, t_{i+1}\right) \mid \mathcal{F}_{t_{i}}\right] \\
= & \lambda_{V} \int_{t_{i}}^{t_{i+1}} e^{r\left(t_{i+1}-t\right)} E^{P}\left[\frac{\partial c^{S V}}{\partial v}\left(t, S_{t}, V_{t}\right) \sigma_{V} V_{t} \mid \mathcal{F}_{t_{i}}\right] d t \\
& +(\mu-r)^{2} \int_{t_{i}}^{t_{i+1}} e^{r\left(t_{i+1}-t\right)} E^{P}\left[S_{t} \mid \mathcal{F}_{t_{i}}\right] E^{P^{S}}\left[\int_{t_{i}}^{t} \frac{\partial^{2} c^{S V}}{\partial s^{2}}\left(u, S_{u}, V_{u}\right) S_{u} d u \mid \mathcal{F}_{t_{i}}\right] d t \\
& +\lambda_{V}(\mu-r) \int_{t_{i}}^{t_{i+1}} e^{r\left(t_{i+1}-t\right)} E^{P}\left[S_{t} \mid \mathcal{F}_{t_{i}}\right] E^{P^{S}}\left[\int_{t_{i}}^{t} \frac{\partial^{2} c^{S V}}{\partial s \partial v}\left(u, S_{u}, V_{u}\right) \sigma_{V} V_{u} d u \mid \mathcal{F}_{t_{i}}\right] d t
\end{aligned}
$$

Proof: The result follows directly from Proposition 6 .

The first term in the expression for the EHE under discrete hedging coincides with the EHE in case of continuous trading (see Corollary 2). If the price of the claim is increasing in volatility, like it is the case for a European call option, the sign of this term equals the sign of the market price of volatility risk.

The second and third term arise because the hedge portfolio is not rebalanced continuously so that they represent the discretization error. The second term, which is analogous to the discretization error in the BS model, is positive if the claim price is a convex function of the stock price. The sign of the third term not only depends on the market price of volatility risk and on the equity risk premium, but also on the expected value of the mixed partial derivative of the claim price with respect to volatility and the stock price. This partial derivative is positive for high strike prices and negative for low strike prices. If the equity risk premium is positive, the sign of the third term and the sign of the market price of volatility risk are therefore equal for high exercise prices and opposite for lower ones.

If the market price of volatility risk equals zero, the EHE is

$$
\begin{aligned}
& E^{P}\left[D^{d}\left(t_{i}, t_{i+1}\right) \mid \mathcal{F}_{t_{i}}\right] \\
& \quad=(\mu-r)^{2} \int_{t_{i}}^{t_{i+1}} e^{r\left(t_{i+1}-t\right)} E^{P}\left[S_{t} \mid \mathcal{F}_{t_{i}}\right] E^{P^{S}}\left[\int_{t_{i}}^{t} \frac{\partial^{2} c^{S V}}{\partial s^{2}}\left(u, S_{u}, V_{u}\right) S_{u} d u \mid \mathcal{F}_{t_{i}}\right] d t
\end{aligned}
$$


For a convex claim like the call, the EHE is strictly positive. Again, this causes a problem for the test described in section 2, since it will signal a positive risk premium even in the case when $\lambda_{V}$ is equal to zero. In general, Corollary 3 shows that the sign of the EHE does not necessarily coincide with the sign of the market price of volatility risk. It is therefore not possible to base inferences about the sign of the market price of volatility risk on the EHE.

As one may expect the absolute value of the difference between the EHEs for continuous and discrete trading depends on the length of the time interval between two consecutive trading dates. The discretization error gets the smaller the smaller this time length, i.e. the more often the portfolio is rebalanced.

The expressions for the EHE in the preceding propositions and corollaries are only 'semi-closed', i.e. we cannot simply plug in the parameters of the problem and obtain a numerical output. We therefore calculate the EHE for this and the following scenarios using a Monte Carlo simulation with 10,000 runs over a hedging horizon of one day for every run. The stochastic differential equations for the dynamics of the stock price and the local variance are discretized using a standard Euler scheme. To avoid problems with possibly negative values for the variance and to come closer to a continuous representation of the stochastic differential equations of the state variables we divide the day into 100 steps of length $\Delta t=1 / 36,525$ each. As described in section 2 the hedge portfolio is composed at time $t_{i}$ (which may be taken to be $t=0$ without any loss of generality) by selling the option short, buying the number of stocks indicated by the delta (BS or Heston) and by borrowing the additional necessary funds at the risk-free rate. The hedging error at time $t_{i+1}$, i.e. after one day, is then given by the expression in equation (4).

To increase the accuracy of our estimates we use the expected value of $V$ at the end of the hedging interval as a control variate. We apply a two-sided $t$-test with significance level $\alpha=0.05$ to test the null hypothesis that the mean hedging error is zero, whereas in the cases when the null states a positive or a negative premium we apply a one-sided test. Note that this does not mean that we consider the t-test appropriate. It is merely this test that has been used in papers trying to identify the sign of $\lambda_{V}$ using expected option 
hedging errors, like Bakshi and Kapadia (2003).

The results for the simulation analysis of the discretization error in the Heston model are shown in table 1 which presents the average hedging error for different maturity classes, strike prices, current values of $V$ and risk premia of the stock. The market price of volatility risk is set to zero. For this exercise the hedging interval is chosen to be one week which coincides with the hedging interval used by Coval and Shumway (2001) in their empirical study of option returns. This period length is chosen to allow an analysis of discretization errors under a 'magnifying glass', since we expect the hedging error to be increasing with the duration of the hedge whereas the qualitative properties of the EHE should remain unchanged compared to shorter hedging intervals.

As one can see from the table the EHE is either not significantly different from zero or significantly positive. This phenomenon is similar to the case of discrete hedging in a BS economy where we can conclude from the closed form representation for the discretization error that the EHE is positive for convex claims. In a Heston economy, there is one additional term in the discretization error compared to BS, so that we cannot draw a similar conclusion a priori. As the simulation results for the Heston model show we would also deduce from a simple discrete hedging test that the volatility risk premium is positive when it is truly zero. This mis-interpretation of the test result occurs mostly for a low current volatility of $\sqrt{V_{0}}=0.1$ and a high equity risk premium of $\mu-r=0.2$.

For the shorter hedging interval of one day and the scenarios considered above the mean hedging error is not statistically different from zero any more for the case of a zero volatility risk premium. For a positive or a negative market price of volatility risk there are some scenarios when a test based on the mean hedging error produces a misleading result, but compared to a hedging interval of one week the number of wrong results reduces significantly. However, this should not lead to the general conclusion that the test is valid for daily hedging intervals, since theoretically the signs of the mean hedging error and the volatility premium could still be different, but the variance of the hedging error is so large that the $t$-statistic becomes insignificant. 


\subsubsection{Discretization Error and Model Mis-Specification}

Like in the case of continuous trading, we now consider the hedging error when the hedge ratio is set equal to the Black-Scholes delta, so that

$$
H_{t_{i}} \equiv \frac{\partial c^{B S}}{\partial s}\left(t_{i}, S_{t_{i}}\right)
$$

The volatility used to compute the partial derivative is the implied volatility for which the BS option price is equal to the price under the correct model. We obtain

Proposition 7 (Heston: EHE with model error and discretization error) The true model is Heston and trading takes is discrete. The hedge ratio $H_{t_{i}}$ at time $t_{i}$ is the $B S$ delta based on the implied volatility of the claim. Then the EHE over the interval $\left[t_{i}, t_{i+1}\right]$ is given by

$$
\begin{aligned}
E^{P}[ & \left.D^{d}\left(t_{i}, t_{i+1}\right) \mid \mathcal{F}_{t_{i}}\right] \\
= & \lambda_{V} \int_{t_{i}}^{t_{i+1}} e^{r\left(t_{i+1}-t\right)} E^{P}\left[\frac{\partial c^{S V}}{\partial v}\left(t, S_{t}, V_{t}\right) \sigma_{V} V_{t} \mid \mathcal{F}_{t_{i}}\right] d t \\
& +(\mu-r)^{2} \int_{t_{i}}^{t_{i+1}} e^{r\left(t_{i+1}-t\right)} E^{P}\left[S_{t} \mid \mathcal{F}_{t_{i}}\right] E^{P^{S}}\left[\int_{t_{i}}^{t} \frac{\partial^{2} c^{S V}}{\partial s^{2}}\left(u, S_{u}, V_{u}\right) S_{u} d u \mid \mathcal{F}_{t_{i}}\right] d t \\
& +(\mu-r) \lambda_{V} \int_{t_{i}}^{t_{i+1}} e^{r\left(t_{i+1}-t\right)} E^{P}\left[S_{t} \mid \mathcal{F}_{t_{i}}\right] E^{P^{S}}\left[\int_{t_{i}}^{t} \frac{\partial^{2} c^{S V}}{\partial s \partial v}\left(u, S_{u}, V_{u}\right) \sigma_{V} V_{u} d u \mid \mathcal{F}_{t_{i}}\right] d t \\
& -(\mu-r) \frac{\partial c^{B S}}{\partial \sigma^{B S}}\left(t_{i}, S_{t_{i}}\right) \frac{\partial \sigma^{B S}}{\partial m}\left(t_{i}, M_{t_{i}}\right) \frac{M_{t_{i}}}{S_{t_{i}}} \int_{t_{i}}^{t_{i+1}} e^{r\left(t_{i+1}-t\right)} E^{P}\left[S_{t} \mid \mathcal{F}_{t_{i}}\right] d t .
\end{aligned}
$$

Proof: Equation (16) follows directly from the proof of Proposition 6 and from equation (22) in the appendix for the relation between the BS delta and the Heston delta.

The first three terms in equation (16) represent the EHE for discrete trading if the hedge ratio is the correct partial derivative from the Heston model (see Corollary 3). The fourth term is an additional component due to the use of an incorrect hedge model. Following the arguments for the case of continuous hedging this term is positive if the market price of risk for the stock is positive and if implied volatility is a decreasing function of the strike price. 
The most important result that we can derive from this proposition is that the sign of the EHE is again in general not equal to the sign of the market price of volatility risk so that we cannot rely on the standard test based on expected option hedging errors.

Tables 2, 3, and 4 present the average hedging errors for different maturity classes, strike prices, current values of $V$ and risk premia for the stock generated by the simulation procedure described above. The market price of volatility risk $\lambda_{V}$ is chosen to be $-2,0$, and 2, and the hedging interval is one day. As discussed above for this period length the discretization error can be neglected more or less so that basically all the error of the test procedure is due to the choice of the wrong hedge coefficient.

If the market price of volatility is zero, the mean hedging error is biased upwards as we can see from the entries in table 2 . In almost all of the cases when the hedging error is statistically different from zero, it is significantly positive. This is perfectly in line with our theoretical arguments which say that for a positive market equity risk premium and for a downward sloping smile curve the choice of the BS delta instead of the Heston partial derivative tends to increase the EHE. Therefore the test from section 2 is not valid in general and the user often tends to wrongly conclude that the market price of volatility risk is positive. This effect is the more pronounced the lower the current value of the local variance. Note, however, that not all the wrong conclusions drawn from the test go in the same direction. We observe a few cases when the test actually signals a negative $\lambda_{V}$. This occurs for 3-month options with a strike price of 110 , positive equity risk premia, and a low volatility.

The most critical scenario in terms of the validity of the standard test procedure is of course the situation when the true volatility risk premium is negative. Since average hedging errors under the assumed scenario of a downward sloping smile and a positive equity risk premium tend to be positive the test is likely to indicate a positive or a zero volatility premium whereas it is indeed negative. As becomes clear from table 3 the problem is again especially pronounced for low current volatility and medium or high equity risk premia. Here it might happen frequently that the sign of the market price of volatility risk is not indicated properly by the hedging test. 
The dependence on the equity risk premium and on the current level of volatility can be explained intuitively. Equation (16) shows that the EHE is the sum of the ideal EHE (no discretization error, no model mis-specification), of the discretization error and the model error. The smaller the ideal EHE and the greater the other error terms, the more often the total EHE may have a sign different from the sign of the ideal EHE and therefore different from the sign of the market price of volatility risk. Now the absolute ideal EHE tends to increase in the current volatility, while the error terms increase in the equity risk premium. Putting these arguments together we can see that for a negative market price of volatility risk, the lower the current volatility and the greater the equity risk premium, the more often the test would produce the wrong signal.

As one may expect for a positive volatility premium the EHE is also almost always significantly positive (see table 4). Of course, this is not due to an improved quality of the test per se, but it is an immediate consequence of the fact that average hedging errors tend to be positive here so that the test more often detects a positive volatility risk premium. In two cases, for short-term options away from the money and a zero equity risk premium the average hedging errors are not significantly different from zero so that we would conclude that volatility risk is not priced.

\subsubsection{Can We Correct for Discretization Error?}

We now try to find a hedge ratio $H_{t_{i}}$ for the Heston model for which the sign of the EHE equals the sign of the market price of volatility risk even under discrete trading. For this hedge ratio it must at least be true that the EHE is zero if the market price of volatility risk is zero. This condition can be used to define the mean-zero hedge ratio.

Proposition 8 (Heston: Mean-zero hedge ratio) The true model is Heston and trading is discrete. The mean-zero hedge ratio is

$$
\begin{aligned}
& H_{t_{i}}^{S V, M Z}\left(t_{i+1}\right)=\frac{\partial c^{S V}}{\partial s}\left(t_{i}, S_{t_{i}}, V_{t_{i}}\right) \\
& \quad+\frac{\int_{t_{i}}^{t_{i+1}} e^{-r t} E^{P}\left[S_{t} \mid \mathcal{F}_{t_{i}}\right] E^{P^{S}}\left[\int_{t_{i}}^{t} \frac{\partial^{2} c^{S V}}{\partial s^{2}}\left(u, S_{u}, V_{u}\right) S_{u} d u \mid \mathcal{F}_{t_{i}}\right] d t}{\int_{t_{i}}^{t_{i+1}} e^{-r t} E^{P}\left[S_{t} \mid \mathcal{F}_{t_{i}}\right] d t}(\mu-r) .
\end{aligned}
$$


The EHE over the interval $\left[t_{i}, t_{i+1}\right]$ generated by this hedge ratio is given by

$$
\begin{aligned}
& E^{P}\left[D^{d}\left(t_{i}, t_{i+1}\right) \mid \mathcal{F}_{t_{i}}\right] \\
& =\lambda_{V} \int_{t_{i}}^{t_{i+1}} e^{r\left(t_{i+1}-t\right)} E^{P}\left[\frac{\partial c^{S V}}{\partial v}\left(t, S_{t}, V_{t}\right) \sigma_{V} V_{t} \mid \mathcal{F}_{t_{i}}\right] d t \\
& \quad+(\mu-r) \lambda_{V} \int_{t_{i}}^{t_{i+1}} e^{r\left(t_{i+1}-t\right)} E^{P}\left[S_{t} \mid \mathcal{F}_{t_{i}}\right] E^{P^{S}}\left[\int_{t_{i}}^{t} \frac{\partial^{2} c^{S V}}{\partial s \partial v}\left(u, S_{u}, V_{u}\right) \sigma_{V} V_{u} d u \mid \mathcal{F}_{t_{i}}\right] d t .
\end{aligned}
$$

Proof: If the market price of volatility risk is zero, Proposition 6 gives

$$
\begin{aligned}
E^{P}\left[D^{d}\left(t_{i}, t_{i+1}\right) \mid \mathcal{F}_{t_{i}}\right] & \\
= & (\mu-r)\left(\frac{\partial c^{S V}}{\partial s}\left(t_{i}, S_{t_{i}}, V_{t_{i}}\right)-H_{t_{i}}\right) \int_{t_{i}}^{t_{i+1}} e^{r\left(t_{i+1}-t\right)} E^{P}\left[S_{t} \mid \mathcal{F}_{t_{i}}\right] d t \\
& \quad+(\mu-r)^{2} \int_{t_{i}}^{t_{i+1}} e^{r\left(t_{i+1}-t\right)} E^{P}\left[S_{t} \mid \mathcal{F}_{t_{i}}\right] E^{P^{S}}\left[\int_{t_{i}}^{t} \frac{\partial^{2} c^{S V}}{\partial s^{2}}\left(u, S_{u}, V_{u}\right) S_{u} d u \mid \mathcal{F}_{t_{i}}\right] d t
\end{aligned}
$$

Setting this expression equal to zero and solving for the hedge ratio gives the mean-zero hedge ratio. The EHE then follows from Proposition 6.

As one can see from the expression for the EHE it is indeed equal to zero for a zero market price of volatility risk. Analogous to the case of the BS model the mean-zero hedge ratio is greater than the partial derivative if the equity risk premium is positive and the claim is convex in the value of the underlying.

However, if the market price of volatility risk is not zero, then the EHE generated by the mean-zero hedge ratio will in general be different from zero, and its sign will not in general coincide with the sign of the market price of volatility risk. Again the test will fail. Taken together the results show that it is impossible to construct a test based on the EHE of a SV option pricing model which is able to identify the sign of the market price of volatility risk consistently.

\section{Conclusion}

In this paper we have investigated whether tests based on expected option hedging errors are able to tell the researcher the sign of the volatility risk premium in an economy with 
stochastic volatility. Recent papers use simple BS hedges and the associated hedging errors to draw conclusions about the sign of this risk premium.

In general, the characteristics of the EHE in an option pricing model depend on the non-hedgeable risks. In case of continuous trading the market prices of non-traded risk factors will affect the EHE, whereas for discrete trading and in case of model mis-specification also the market price of equity risk becomes relevant.

The main results of our paper can be summarized as follows. The error introduced by discretization may destroy the properties of tests based on the EHE in that its sign and the sign of market price of volatility risk do not necessarily coincide both in the BS model and under stochastic volatility. An important difference from a theoretical point of view is that for the BS model there is a remedy to this problem if we base the test on what we call the mean-zero hedge ratio instead of the classical delta. However, for the Heston model there is in general no such adjustment so that any test based on discrete hedging will suffer from the problem described above, except in the case of a zero volatility risk premium. Using an incorrect model (like BS in the case of stochastic volatility) to set up the hedge also leads to a test with unreliable properties, even in the case of continuous trading. Especially when the true data generating process exhibits low volatility relative to the long-term mean the test quite frequently gives a wrong indication for the sign of the volatility risk premium.

The analyzes performed in this paper constitute a first step towards a more detailed investigation of the characteristics of option hedging errors. A first step in this direction might be to search for more robust tests, which are less sensitive to discretization error and model mis-specification. For example, one might consider hedge portfolios consisting of two (or more) options instead of options and the stock to perform the hedging test. Furthermore a researcher might also be confronted with a model identification problem. Are hedge results actually generated by volatility risk premia? Is it possible that other risk factors are present, like jump risk and the risk associated with uncertain jump sizes? Especially the inclusion of these types of risk seems a natural next step towards a better understanding of the relationship between hedging errors and risk premia. 


\section{References}

Bakshi, G., C. Cao, and Z. Chen (1997) Empirical Performance of Alternative Option Pricing Models, Journal of Finance 52, 2003-2049.

Bakshi, G., and N. Kapadia (2003) Delta-Hedged Gains and the Negative Market Volatility Risk Premium, .

Bergman, Y. Z., B. D. Grundy, and Z. Wiener (1996) General Properties of Option Prices, Journal of Finance 51, 1573-1610.

Bertsimas, D., L. Kogan, and A. W. Lo (2000) When is Time Continuous?, Journal of Financial Economics 55, 173-204.

Black, F., and M. Scholes (1973) The Pricing of Options and Corporate Liabilities, Journal of Political Economy 81, 637-654.

Boyle, P. P., and D. Emanuel (1980) Discretly Adjusted Option Hedges, Journal of Financial Economics 8, 259-282.

Buraschi, A., and J. Jackwerth (2001) The Price of a Smile: Hedging and Spanning in Option Markets, Review of Financial Studies 14, 495-527.

Coval, J. D., and T. Shumway (2001) Expected Options Returns, Journal of Finance 56, 983-1009.

Dudenhausen, A. (2002) Effectiveness of hedging strategies under model misspecification and trading restrictions, Working Paper.

Heston, S. L. (1993) A closed-form solution for options with stochastic volatility with applications to bond and currency options, Review of Financial Studies 6, 327-343.

Rubinstein, M. (1984) A Simple Formula for the Expected Rate of Return of an Option Over a Finite Holding Period, Journal of Finance 39, 1503-1509.

Schöbel, R., and J. Zhu (1999) Stochastic Volatility With an Ornstein-Uhlenbeck Process: An Extension, European Finance Review 3, 23-46. 


\section{A Appendix}

\section{A.1 Proof of Proposition 2}

In case of discrete trading, the hedging error over the interval $\left[t_{i}, t_{i+1}\right]$ is

$$
D^{d}\left(t_{i}, t_{i+1}\right)=\int_{t_{i}}^{t_{i+1}} e^{r\left(t_{i+1}-t\right)}\left(\frac{\partial c^{B S}}{\partial s}\left(t, S_{t}\right)-H_{t_{i}}\right)\left(d S_{t}-r S_{t} d t\right)
$$

where we have used expression (7) for the change in the price of the claim.

Taking expectations yields

$$
\begin{aligned}
E^{P} & {\left[D^{d}\left(t_{i}, t_{i+1}\right) \mid \mathcal{F}_{t_{i}}\right] } \\
& =E^{P}\left[\int_{t_{i}}^{t_{i+1}} e^{r\left(t_{i+1}-t\right)}\left(\frac{\partial c^{B S}}{\partial s}\left(t, S_{t}\right)-H_{t_{i}}\right)(\mu-r) S_{t} d t \mid \mathcal{F}_{t_{i}}\right] \\
& =(\mu-r) \int_{t_{i}}^{t_{i+1}} e^{r\left(t_{i+1}-t\right)} E^{P}\left[S_{t} \mid \mathcal{F}_{t_{i}}\right] E^{P^{S(t)}}\left[\frac{\partial c^{B S}}{\partial s}\left(t, S_{t}\right)-H_{t_{i}} \mid \mathcal{F}_{t_{i}}\right] d t .
\end{aligned}
$$

The probability measure $P^{S}$ is defined by the Radon-Nikodym derivative

$$
\frac{d P^{S}}{d P}=\frac{S_{T}}{E^{P}\left[S_{T}\right]}
$$

The expected hedging error depends on the difference between the partial derivatives in the interval from $t_{i}$ to $t_{i+1}$ and the fixed hedge ratio $H_{t_{i}}$. We further decompose these terms into the difference between the partial derivative at time $t_{i}$ and the hedge ratio used, and into the change of the partial derivative in the interval from $t_{i}$ to $t_{i+1}$. Applying Ito to the hedge ratio gives

$$
\begin{aligned}
& \frac{\partial c^{B S}}{\partial s}\left(t, S_{t}\right)=\frac{\partial c^{B S}}{\partial s}\left(t_{i}, S_{t_{i}}\right) \\
& \quad+\int_{t_{i}}^{t}\left(\frac{\partial^{2} c^{B S}}{\partial t \partial s}\left(u, S_{u}\right) d u+\frac{\partial^{2} c^{B S}}{\partial s^{2}}\left(u, S_{u}\right) d S_{u}+\frac{1}{2} \frac{\partial^{3} c^{B S}}{\partial s^{3}}\left(u, S_{u}\right) \sigma^{2} S_{u}^{2} d u\right) .
\end{aligned}
$$

Differentiating the fundamental partial differential equation once with respect to the stock price gives

$$
\begin{aligned}
& \frac{\partial^{2} c^{B S}}{\partial t \partial s}\left(u, S_{u}\right)+\frac{\partial^{2} c^{B S}}{\partial s^{2}}\left(u, S_{u}\right) r S_{u}+\frac{\partial c^{B S}}{\partial s}\left(u, S_{u}\right) r \\
& \quad+\frac{1}{2} \frac{\partial^{3} c^{B S}}{\partial s^{3}}\left(u, S_{u}\right) \sigma^{2} S_{u}^{2}+\frac{\partial^{2} c^{B S}}{\partial s^{2}}\left(u, S_{u}\right) \sigma^{2} S_{u}-r \frac{\partial c^{B S}}{\partial s}\left(u, S_{u}\right)=0
\end{aligned}
$$


so that the difference between the hedge ratios at time $t$ and at time $t_{i}$ is

$$
\frac{\partial c^{B S}}{\partial s}\left(t, S_{t}\right)-\frac{\partial c^{B S}}{\partial s}\left(t_{i}, S_{t_{i}}\right)=\int_{t_{i}}^{t} \frac{\partial^{2} c^{B S}}{\partial s^{2}}\left(u, S_{u}\right)\left(d S_{u}-\left(r+\sigma^{2}\right) S_{u} d u\right) .
$$

The expected hedging error from equation (17) can therefore be written as

$$
\begin{gathered}
E^{P}\left[D\left(t_{i}, t_{i+1}\right) \mid \mathcal{F}_{t_{i}}\right] \\
=(\mu-r) \int_{t_{i}}^{t_{i+1}} e^{r\left(t_{i+1}-t\right)} E^{P}\left[S_{t} \mid \mathcal{F}_{t_{i}}\right] \\
E^{P^{S(t)}}\left[\frac{\partial c^{B S}}{\partial s}\left(t_{i}, S_{t_{i}}\right)\right. \\
\left.\quad+\int_{t_{i}}^{t} \frac{\partial^{2} c^{B S}}{\partial s^{2}}\left(u, S_{u}\right)\left(d S_{u}-\left(r+\sigma^{2}\right) S_{u} d u\right)-H_{t_{i}} \mid \mathcal{F}_{t_{i}}\right] d t \\
=(\mu-r)\left(\frac{\partial c^{B S}}{\partial s}\left(t_{i}, S_{t_{i}}\right)-H_{t_{i}}\right) \int_{t_{i}}^{t_{i+1}} e^{r\left(t_{i+1}-t\right)} E^{P}\left[S_{t} \mid \mathcal{F}_{t_{i}}\right] d t \\
+(\mu-r) \int_{t_{i}}^{t_{i+1}} e^{r\left(t_{i+1}-t\right)} E^{P}\left[S_{t} \mid \mathcal{F}_{t_{i}}\right] \\
E^{P^{S}}\left[\int_{t_{i}}^{t} \frac{\partial^{2} c^{B S}}{\partial s^{2}}\left(u, S_{u}\right)\left(d S_{u}-\left(r+\sigma^{2}\right) S_{u} d u\right) \mid \mathcal{F}_{t_{i}}\right] d t .
\end{gathered}
$$

Noting that under $P^{S}$ the drift of the stock is equal to $\mu+\sigma^{2}$ we obtain the proposition.

\section{A.2 Proof of Proposition 4}

Under the usual mild technical conditions, the dynamics of any derivative contract in our SV model are given by

$$
\begin{aligned}
d C_{t}^{S V}= & \frac{\partial c^{S V}}{\partial t}\left(t, S_{t}, V_{t}\right) d t+\frac{\partial c^{S V}}{\partial s}\left(t, S_{t}, V_{t}\right) d S_{t}+\frac{\partial c^{S V}}{\partial v}\left(t, S_{t}, V_{t}\right) d V_{t} \\
& +\frac{1}{2} \frac{\partial^{2} c^{S V}}{\partial s^{2}}\left(t, S_{t}, V_{t}\right) V_{t} S_{t}^{2} d t+\frac{1}{2} \frac{\partial^{2} c^{S V}}{\partial v^{2}}\left(t, S_{t}, V_{t}\right) \sigma_{V}^{2} V_{t} d t \\
& +\frac{1}{2} \frac{\partial^{2} c^{S V}}{\partial s \partial v}\left(t, S_{t}, V_{t}\right) V_{t} S_{t} \sigma_{V} \rho d t \\
= & r C_{t}^{S V} d t+\frac{\partial c^{S V}}{\partial s}\left(t, S_{t}, V_{t}\right)\left(d S_{t}-r S_{t} d t\right) \\
& +\frac{\partial c^{S V}}{\partial v}\left(t, S_{t}, V_{t}\right)\left(d V_{t}-\kappa\left(\theta-V_{t}\right) d t+\lambda_{V} \sigma_{V} V_{t} d t\right)
\end{aligned}
$$


where the second equality follows from the well-known fundamental partial differential equation that the pricing function for every path-independent claim has to satisfy.

Plugging equation (19) for the change in the price of the derivative into the general formula (3) for the hedging error gives

$$
\begin{aligned}
D^{c}(t, t+\tau)= & \int_{t}^{t+\tau} e^{r(t+\tau-u)}\left(\frac{\partial c^{S V}}{\partial s}\left(u, S_{u}, V_{u}\right)-H_{u}\right)\left(d S_{u}-r S_{u} d u\right) \\
& +\int_{t}^{t+\tau} e^{r(t+\tau-u)} \frac{\partial c^{S V}}{\partial v}\left(u, S_{u}, V_{u}\right)\left(d V_{u}-\kappa\left(\theta-V_{u}\right) d u+\lambda_{V} \sigma_{V} V_{u} d u\right) .
\end{aligned}
$$

Taking expectations gives the proposition.

\section{A.3 Proof of Proposition 5}

From Proposition 4 we get

$$
\begin{aligned}
& E^{P}\left[D^{c}(t, t+\tau) \mid \mathcal{F}_{t}\right] \\
& =\lambda_{V} \int_{t}^{t+\tau} e^{r(t+\tau-u)} E^{P}\left[\frac{\partial c^{S V}}{\partial v}\left(u, S_{u}, V_{u}\right) \sigma_{V} V_{u} \mid \mathcal{F}_{t}\right] d u \\
& \quad+(\mu-r) \int_{t}^{t+\tau} e^{r(t+\tau-u)} E^{P}\left[\left(\frac{\partial c^{S V}}{\partial s}\left(u, S_{u}, V_{u}\right)-\frac{\partial c^{B S}}{\partial s}\left(u, S_{u}\right)\right) S_{u} \mid \mathcal{F}_{t}\right] d u .(21
\end{aligned}
$$

The first term is the EHE when the hedge ratio is the partial derivative. It is given in Corollary 2 , and its sign equals the sign of the market price of volatility risk.

To analyze the difference between the partial derivatives from the BS and the Heston model we start from the definition of the implied volatility:

$$
c^{B S}\left(t, s ; \sigma^{B S}\right)=c^{S V}(t, s, v) .
$$

where we introduce volatility as an additional argument in the BS price. Differentiating both sides once with respect to the stock price gives

$$
\frac{\partial c^{B S}}{\partial s}(t, s)+\frac{\partial c^{B S}}{\partial \sigma}(t, s) \cdot \frac{\partial \sigma^{B S}}{\partial s}(t, s)=\frac{\partial c^{S V}}{\partial s}(t, s, v)
$$

so that the difference between the two hedge ratios is

$$
\frac{\partial c^{S V}}{\partial s}(t, s, v)-\frac{\partial c^{B S}}{\partial s}(t, s)=\frac{\partial c^{B S}}{\partial \sigma}(t, s) \cdot \frac{\partial \sigma^{B S}}{\partial s}(t, s) .
$$


The first factor of the product on the right-hand side is always positive, the sign of the second factor depends on the slope of the volatility smile. It can be simplified by noting that in the BS model the call price is a positive homogeneous function of the stock price and the strike so that

$$
\sigma^{B S}(t, s, K)=\sigma^{B S}(t, 1, m)
$$

where the moneyness $m$ is defined as $m=\frac{K}{s}$. In the following, we therefore write $\sigma^{B S}$ as a function of time $t$ and moneyness $m$. This implies

$$
\frac{\partial \sigma^{B S}}{\partial s}(t, s)=-\frac{\partial \sigma^{B S}}{\partial m}(t, m) \cdot \frac{m}{s} .
$$

This gives

$$
\frac{\partial c^{S V}}{\partial s}(t, s, v)-\frac{\partial c^{B S}}{\partial s}(t, s)=-\frac{\partial c^{B S}}{\partial \sigma}(t, s) \cdot \frac{\partial \sigma^{B S}}{\partial m}(t, m) \frac{m}{s}
$$

which together with (21) yields the proposition.

\section{A.4 Proof of Proposition 6}

In case of discrete trading, the hedging error (4) over the interval $\left[t_{i}, t_{i+1}\right]$ is given by

$$
\begin{aligned}
D^{d}\left(t_{i}, t_{i+1}\right)= & \left(\int_{t_{i}}^{t_{i+1}} e^{r\left(t_{i+1}-u\right)}\left(d C_{u}-r C_{u} d u\right)-\int_{t_{i}}^{t_{i+1}} e^{-r u} H_{t_{i}}\left(d S_{u}-r S_{u} d u\right)\right) \\
= & \int_{t_{i}}^{t_{i+1}} e^{r\left(t_{i+1}-u\right)}\left(\frac{\partial c^{S V}}{\partial s}\left(t, S_{t}, V_{t}\right)-H_{t_{i}}\right)\left(d S_{t}-r S_{t} d t\right) \\
& +\int_{t_{i}}^{t_{i+1}} e^{r\left(t_{i+1}-u\right)} \frac{\partial c^{S V}}{\partial v}\left(t, S_{t}, V_{t}\right)\left(d V_{t}-\left(\kappa\left(\theta-V_{t}\right)-\lambda_{V} \sigma_{V} V_{t}\right) d t\right)
\end{aligned}
$$


where we have used expression (19) for the change in the price of the claim to obtain the second equality. The expected hedging error is now equal to

$$
\begin{aligned}
E^{P}[ & \left.D^{d}\left(t_{i}, t_{i+1}\right) \mid \mathcal{F}_{t_{i}}\right] \\
= & E^{P}\left[\int_{t_{i}}^{t_{i+1}} e^{r\left(t_{i+1}-t\right)}\left(\frac{\partial c^{S V}}{\partial s}\left(t, S_{t}, V_{t}\right)-H_{t_{i}}\right)(\mu-r) S_{t} \mid \mathcal{F}_{t_{i}}\right] \\
& +E^{P}\left[\int_{t_{i}}^{t_{i+1}} e^{r\left(t_{i+1}-t\right)} \frac{\partial c^{S V}}{\partial s}\left(t, S_{t}, V_{t}\right) \lambda_{V} \sigma_{V} V_{t} d t \mid \mathcal{F}_{t_{i}}\right] \\
= & \lambda_{V} E^{P}\left[\int_{t_{i}}^{t_{i+1}} e^{r\left(t_{i+1}-t\right)} \frac{\partial c^{S V}}{\partial v}\left(t, S_{t}, V_{t}\right) \sigma_{V} V_{t} d t \mid \mathcal{F}_{t_{i}}\right] \\
& +(\mu-r) \int_{t_{i}}^{t_{i+1}} e^{r\left(t_{i+1}-t\right)} E^{P}\left[S_{t} \mid \mathcal{F}_{t_{i}}\right] E^{P^{S(t)}}\left[\frac{\partial c^{S V}}{\partial s}\left(t, S_{t}, V_{t}\right)-H_{t_{i}} \mid \mathcal{F}_{t_{i}}\right] d t .
\end{aligned}
$$

We now take a closer look at the partial derivative at time $t$. Applying Ito gives

$$
\begin{aligned}
\frac{\partial c^{S V}}{\partial s}\left(t, S_{t}, V_{t}\right)= & \frac{\partial c^{S V}}{\partial s}\left(t_{i},\right. \\
& \left.S_{t_{i}}, V_{t_{i}}\right) \\
& +\int_{t_{i}}^{t}\left(\frac{\partial^{2} c^{S V}}{\partial t \partial s}\left(u, S_{u}, V_{u}\right) d u+\frac{\partial^{2} c^{S V}}{\partial s^{2}}\left(u, S_{u}, V_{u}\right) d S_{u}\right. \\
& +\frac{\partial^{2} c^{S V}}{\partial s \partial v}\left(u, S_{u}, V_{u}\right) d V_{u}+\frac{1}{2} \frac{\partial^{3} c^{S V}}{\partial s^{3}}\left(u, S_{u}, V_{u}\right) V_{u} S_{u}^{2} d u \\
& \left.+\frac{1}{2} \frac{\partial^{3} c^{S V}}{\partial s \partial v^{2}}\left(u, S_{u}, V_{u}\right) \sigma_{V}^{2} V_{u} d u+\frac{\partial^{3} c^{S V}}{\partial s^{2} \partial v}\left(u, S_{u}, V_{u}\right) \sigma_{V} \rho S_{u} V_{u} d u\right) .
\end{aligned}
$$

Differentiating the fundamental partial differential equation once with respect to the stock price gives

$$
\begin{gathered}
\frac{\partial^{2} c^{S V}}{\partial t \partial s}\left(u, S_{u}, V_{u}\right)+\frac{\partial^{2} c^{S V}}{\partial s^{2}}\left(u, S_{u}, V_{u}\right) r S_{u}+\frac{\partial c^{S V}}{\partial s}\left(u, S_{u}, V_{u}\right) r \\
+\frac{\partial^{2} c^{S V}}{\partial s \partial v}\left(u, S_{u}, V_{u}\right)\left(\kappa\left(\theta-V_{u}\right)-\lambda_{V} \sigma_{V} V_{u}\right)+\frac{1}{2} \frac{\partial^{3} c^{S V}}{\partial s^{3}}\left(u, S_{u}, V_{u}\right) V_{u} S_{u}^{2} \\
+\frac{\partial^{2} c^{S V}}{\partial s^{2}}\left(u, S_{u}, V_{u}\right) V_{u} S_{u}+\frac{1}{2} \frac{\partial^{3} c^{S V}}{\partial s \partial v^{2}}\left(u, S_{u}, V_{u}\right) \sigma_{V}^{2} V_{u} \\
+\frac{\partial^{3} c^{S V}}{\partial s^{2} \partial v}\left(u, S_{u}, V_{u}\right) \sigma_{V} \rho S_{u} V_{u}+\frac{\partial^{2} c^{S V}}{\partial s \partial v}\left(u, S_{u}, V_{u}\right) \sigma_{V} \rho V_{u}-r \frac{\partial c^{S V}}{\partial s}\left(u, S_{u}, V_{u}\right)=0
\end{gathered}
$$

so that the difference between the hedge ratio at time $t$ and time $t_{i}$ is

$$
\begin{aligned}
& \frac{\partial c^{S V}}{\partial s}\left(t, S_{t}, V_{t}\right)-\frac{\partial c^{S V}}{\partial s}\left(t_{i}, S_{t_{i}}, V_{t_{i}}\right) \\
& =\int_{t_{i}}^{t} \frac{\partial^{2} c}{\partial s^{2}}\left(u, S_{u}, V_{u}\right)\left(d S_{u}-\left(r+V_{u}\right) S_{u} d u\right) \\
& \quad+\int_{t_{i}}^{t} \frac{\partial^{2} c^{S V}}{\partial s \partial v}\left(u, S_{u}, V_{u}\right)\left(d V_{u}-\kappa\left(\theta-V_{u}\right) d u+\lambda_{V} \sigma_{V} V_{u} d u-\sigma_{V} \rho V_{u} d u\right) .
\end{aligned}
$$


Plugging this expression into the equation for the expected hedging error (23) gives

$$
\begin{aligned}
& E^{P}\left[D^{d}\left(t_{i}, t_{i+1}\right) \mid \mathcal{F}_{t_{i}}\right] \\
& =\lambda_{V} E^{P}\left[\int_{t_{i}}^{t_{i+1}} e^{r\left(t_{i+1}-t\right)} \frac{\partial c^{S V}}{\partial v}\left(t, S_{t}, V_{t}\right) \sigma_{V} V_{t} d t \mid \mathcal{F}_{t_{i}}\right] \\
& \quad+(\mu-r)\left(\frac{\partial c^{S V}}{\partial s}\left(t_{i}, S_{t_{i}}, V_{t_{i}}\right)-H_{t_{i}}\right) \int_{t_{i}}^{t_{i+1}} e^{r\left(t_{i+1}-t\right)} E^{P}\left[S_{t} \mid \mathcal{F}_{t_{i}}\right] d t \\
& \quad+(\mu-r) \int_{t_{i}}^{t_{i+1}} e^{r\left(t_{i+1}-t\right)} E^{P}\left[S_{t} \mid \mathcal{F}_{t_{i}}\right] \\
& \quad E^{P^{S}}\left[\int_{t_{i}}^{t} \frac{\partial^{2} c^{S V}}{\partial s^{2}}\left(u, S_{u}, V_{u}\right)\left(d S_{u}-\left(r+V_{u}\right) S_{u} d u\right) \mid \mathcal{F}_{t_{i}}\right] d t \\
& \quad+(\mu-r) \int_{t_{i}}^{t_{i+1}} e^{r\left(t_{i+1}-t\right)} E^{P}\left[S_{t} \mid \mathcal{F}_{t_{i}}\right] \\
& E^{P^{S}}\left[\int_{t_{i}}^{t} \frac{\partial^{2} c^{S V}}{\partial s \partial v}\left(u, S_{u}, V_{u}\right)\right. \\
& \left.\quad\left(d V_{u}-\kappa\left(\theta-V_{u}\right) d u+\lambda_{V} \sigma_{V} V_{u} d u-\sigma_{V} \rho V_{u} d u\right) \mid \mathcal{F}_{t_{i}}\right] d t .
\end{aligned}
$$

Under $P^{S}$, the drift of the stock price is equal to $\mu+V$ and the drift of $V$ is equal to $\kappa(\theta-V)+\sigma_{V} \rho V$. This yields the proposition. 
Table 1: Impact of discretization error in the Heston model $\left(\lambda_{V}=0\right)$

Mean hedging error (multiplied by 1,000) for a European call option as a function of the strike $K$, the equity risk premium $\mu-r$, and time to maturity $T T M$ in months. The hedge ratio is given by the Heston delta, the hedging interval is one week $(\tau-t=1 / 52)$. The parameters of the risk-neutral process are given by $S_{0}=100, r=0.05, \kappa_{q}=1.15, \theta_{q}=0.04, \sigma_{v}=0.4, \rho=-0.65$.

' + ' behind a mean hedging error entry indicates that it is significantly positive. Based on a two sided $t$-test one would thus wrongly reject the null hypothesis of a zero market price of volatility risk.

\begin{tabular}{|c|c|c|c|c|c|c|c|c|c|c|}
\hline \multirow[b]{2}{*}{$T T M$} & \multirow[b]{2}{*}{$\mu-r$} & \multicolumn{3}{|c|}{$V_{0}=0.01$} & \multicolumn{3}{|c|}{$V_{0}=0.04$} & \multicolumn{3}{|c|}{$V_{0}=0.09$} \\
\hline & & $K=90$ & $K=100$ & $K=110$ & $K=90$ & $K=100$ & $K=110$ & $K=90$ & $K=100$ & $K=110$ \\
\hline 1 & 0 & -0.069 & -2.181 & -0.001 & -1.257 & -3.485 & -0.255 & $\mid-3.368$ & -4.757 & -1.598 \\
\hline 3 & 0 & -0.260 & -1.337 & -0.040 & -1.145 & -2.311 & -1.267 & -2.245 & -3.114 & -2.523 \\
\hline 6 & 0 & -0.281 & -0.850 & -0.386 & -0.928 & -1.740 & -1.624 & -1.705 & -2.403 & -2.471 \\
\hline 12 & 0 & -0.228 & -0.483 & -0.617 & -0.707 & -1.204 & -1.476 & -1.290 & -1.829 & -2.139 \\
\hline 1 & 0.1 & -0.027 & -0.081 & 0.005 & -1.040 & -2.436 & 0.033 & -3.067 & -4.135 & -1.212 \\
\hline 3 & 0.1 & -0.117 & -0.275 & 0.248 & -0.910 & -1.698 & -0.664 & -2.012 & -2.728 & -2.126 \\
\hline 6 & 0.1 & -0.123 & -0.209 & 0.480 & -0.736 & -1.318 & -1.032 & -1.526 & -2.123 & -2.133 \\
\hline 12 & 0.1 & -0.086 & -0.116 & 0.103 & -0.563 & -0.926 & -1.041 & -1.160 & -1.628 & -1.874 \\
\hline 1 & 0.2 & 0.087 & $6.350+$ & $0.020+$ & -0.442 & 1.098 & 1.092 & -2.142 & -1.811 & 0.351 \\
\hline 3 & 0.2 & 0.306 & $2.934+$ & $1.192+$ & -0.176 & 0.303 & 1.464 & -1.223 & -1.352 & -0.629 \\
\hline 6 & 0.2 & 0.355 & $1.733+$ & $3.258+$ & -0.123 & 0.046 & 0.987 & -0.912 & -1.138 & -0.896 \\
\hline 12 & 0.2 & 0.354 & $1.007+$ & $2.339+$ & -0.094 & -0.022 & 0.400 & $\mid-0.706$ & -0.926 & -0.927 \\
\hline
\end{tabular}


Table 2: Impact of discretization error and model mis-specification in the Heston model $\left(\lambda_{V}=0\right)$

Mean hedging error (multiplied by 1,000) for a European call option as a function of the strike $K$, of the equity risk premium $\mu-r$, and of the time to maturity TTM in months. The hedge ratio is given by the Heston delta, the hedging interval is one day $(\tau-t=1 / 365)$, and the hedge is not rebalanced in between.. The parameters of the risk-neutral process are given by $S_{0}=100, r=0.05, \kappa_{q}=1.15, \theta_{q}=0.04, \sigma_{v}=0.4, \rho=-0.65$.

' + '('-') behind the mean hedging error entry indicates that it is significantly positive (negative). Based on a two-sided $t$-test one would thus wrongly reject the null hypothesis of a zero market price of volatility risk.

\begin{tabular}{|c|c|c|c|c|c|c|c|c|c|c|c|c|c|c|c|c|c|c|c|}
\hline \multirow{3}{*}{$\begin{array}{r}T T M \\
1\end{array}$} & \multirow[b]{2}{*}{$\mu-r$} & \multicolumn{6}{|c|}{$V_{0}=0.01$} & \multicolumn{6}{|c|}{$V_{0}=0.04$} & \multicolumn{6}{|c|}{$V_{0}=0.09$} \\
\hline & & \multicolumn{2}{|c|}{$K=90$} & \multicolumn{2}{|c|}{$K=100$} & \multicolumn{2}{|c|}{$K=110$} & \multicolumn{2}{|c|}{$K=90$} & \multicolumn{2}{|c|}{$K=100$} & \multicolumn{2}{|c|}{$K=110$} & \multicolumn{2}{|c|}{$K=90$} & \multicolumn{2}{|c|}{$K=100$} & \multicolumn{2}{|l|}{$K=110$} \\
\hline & 0 & -0.023 & & -0.277 & & 0.000 & & -0.177 & & -0.590 & & -0.093 & & -0.454 & & -0.892 & & -0.456 & \\
\hline 3 & 0 & -0.073 & & -0.169 & & -0.013 & & -0.236 & & -0.370 & & -0.190 & & -0.424 & & -0.558 & & -0.448 & \\
\hline 6 & 0 & -0.077 & & -0.131 & & -0.041 & & -0.214 & & -0.290 & & -0.217 & & -0.360 & & -0.440 & & -0.406 & \\
\hline 12 & 0 & -0.071 & & -0.105 & & -0.091 & & -0.182 & & -0.235 & & -0.227 & & -0.304 & & -0.366 & & -0.373 & \\
\hline 1 & 0.1 & 0.029 & & 1.644 & + & 0.004 & + & 0.040 & & 0.445 & & 0.046 & & -0.148 & & -0.195 & & -0.090 & \\
\hline 3 & 0.1 & 0.514 & + & 2.380 & + & -0.080 & - & 0.579 & & 1.315 & + & 0.635 & + & 0.361 & & 0.615 & & 0.546 & \\
\hline 6 & 0.1 & 1.067 & + & 2.534 & + & 0.515 & + & 1.084 & + & 1.835 & + & 1.373 & + & 0.816 & & 1.149 & & 1.123 & \\
\hline 12 & 0.1 & 1.483 & + & 2.449 & + & 2.127 & + & 1.480 & + & 2.096 & + & 2.038 & + & 1.241 & & 1.591 & & 1.644 & \\
\hline 1 & 0.2 & 0.083 & + & 3.658 & + & 0.010 & + & 0.267 & & 1.531 & + & 0.200 & & 0.172 & & 0.536 & & 0.300 & \\
\hline 3 & 0.2 & 1.107 & + & 4.974 & + & -0.134 & - & 1.404 & + & 3.030 & + & 1.492 & + & 1.158 & + & 1.809 & + & $1.563+$ & + \\
\hline 6 & 0.2 & 2.218 & + & 5.228 & + & 1.108 & + & 2.390 & + & 3.980 & + & 2.992 & + & 2.002 & + & 2.752 & + & $2.671+$ & + \\
\hline 12 & 0.2 & 3.043 & + & 5.020 & + & 4.377 & + & 3.149 & + & 4.440 & + & 4.325 & + & 2.792 & + & 3.558 & + & $3.676+$ & + \\
\hline
\end{tabular}


Table 3: Impact of discretization error and model mis-specification in the Heston model $\left(\lambda_{V}=-2\right)$

Mean hedging error (multiplied by 1,000) for a European call option as a function of the strike $K$, of the equity risk premium $\mu-r$, and of the time to maturity TTM in month. The hedge ratio is given by the Heston delta, the hedging interval is one day $8 \tau-t=1 / 365)$, and the hedge is not rebalanced in between.. The parameters of the risk-neutral process are given by $S_{0}=100, r=0.05, \kappa_{q}=1.15, \theta_{q}=0.04, \sigma_{v}=0.4, \rho=-0.65$.

' + ' ('0') behind the mean hedging error indicates that it is significantly positive (not significantly different from zero). Based on a one-sided $t$-test one would thus wrongly reject the null hypothesis of a negative market price of volatility risk.

\begin{tabular}{|c|c|c|c|c|c|c|c|c|c|c|c|c|c|c|}
\hline \multirow[b]{2}{*}{ TTM } & \multirow[b]{2}{*}{$\mu-r$} & \multicolumn{6}{|c|}{$V_{0}=0.01$} & \multicolumn{4}{|c|}{$V_{0}=0.04$} & \multicolumn{3}{|c|}{$V_{0}=0.09$} \\
\hline & & $K=9$ & & $K=1$ & & $K=1$ & & $K=9$ & & $K=100$ & $K=110$ & $K=90$ & $K=100$ & $K=110$ \\
\hline 1 & 0 & -0.063 & & -1.386 & & -0.002 & 0 & -0.679 & & -2.947 & -0.567 & -1.997 & -4.467 & -2.511 \\
\hline 3 & 0 & -0.482 & & -1.707 & & -0.241 & & -2.053 & & -4.097 & -2.799 & -4.237 & -6.349 & -5.798 \\
\hline 6 & 0 & -0.843 & & -1.747 & & -1.031 & & -2.978 & & -4.798 & -4.533 & $\mid-5.700$ & -7.745 & -8.026 \\
\hline 12 & 0 & -1.007 & & -1.562 & & -1.643 & & -3.489 & & -4.901 & -5.366 & -6.649 & -8.549 & -9.409 \\
\hline 1 & 0.1 & -0.010 & 0 & 0.538 & 0 & 0.003 & + & -0.458 & & -1.911 & -0.433 & -1.685 & -3.769 & -2.154 \\
\hline 3 & 0.1 & 0.107 & 0 & 0.844 & + & -0.310 & & -1.234 & & -2.409 & -1.984 & -3.447 & -5.174 & -4.811 \\
\hline 6 & 0.1 & 0.302 & 0 & 0.920 & + & -0.481 & & -1.677 & & -2.671 & -2.950 & -4.519 & -6.155 & -6.502 \\
\hline 12 & 0.1 & 0.548 & + & 0.993 & + & 0.573 & 0 & -1.824 & & -2.568 & -3.103 & $\mid-5.101$ & -6.590 & -7.394 \\
\hline 1 & 0.2 & 0.044 & + & 2.554 & + & 0.008 & + & -0.229 & & -0.823 & -0.285 & -1.361 & -3.037 & -1.773 \\
\hline 3 & 0.2 & 0.701 & + & 3.441 & + & -0.367 & & -0.405 & J & $-0.693 \quad c$ & -1.138 & -2.645 & -3.979 & -3.802 \\
\hline 6 & 0.2 & 1.455 & + & 3.615 & + & 0.106 & 0 & -0.367 & & $-0.524 \quad \mathrm{C}$ & -1.339 & -3.330 & -4.550 & -4.959 \\
\hline 12 & 0.2 & 2.110 & + & 3.564 & + & 2.821 & + & -0.153 & o & $-0.221 \mathrm{c}$ & $-0.819 \quad \mathbf{0}$ & -3.546 & -4.621 & -5.365 \\
\hline
\end{tabular}


Table 4: Impact of discretization error and model mis-specification in the Heston model $\left(\lambda_{V}=2\right)$

Mean hedging error (multiplied by 1,000) for a European call option as a function of the strike $K$, the equity risk premium $\mu-r$, and the time to maturity TTM in months. The hedge ratio is given by the Heston delta, the hedging interval is one day $(\tau-t=1 / 365)$, and the hedge is not rebalanced in between. The parameters of the risk-neutral process are given by $S_{0}=100, r=0.05, \kappa_{q}=1.15, \theta_{q}=0.04, \sigma_{v}=0.4, \rho=-0.65$.

'0' behind the mean hedging error entry indicates that it is not significantly different from zero. Based on a one-sided $t$-test one would thus wrongly reject the hypothesis of a positive market price of volatility risk.

\begin{tabular}{|c|c|c|c|c|c|c|c|c|c|c|}
\hline \multirow[b]{2}{*}{ TTM } & \multirow[b]{2}{*}{$\mu-r$} & \multicolumn{3}{|c|}{$V_{0}=0.01$} & \multicolumn{3}{|c|}{$V_{0}=0.04$} & \multicolumn{3}{|c|}{$V_{0}=0.09$} \\
\hline & & $K=90$ & $K=100$ & $K=110$ & $K=90$ & $K=100$ & $K=110$ & $K=90$ & $K=100$ & $K=110$ \\
\hline 1 & 0 & $0.017 \quad \mathbf{0}$ & 0.833 & $0.001 \quad \mathbf{0}$ & 0.326 & 1.770 & 0.383 & 1.093 & 2.687 & 1.604 \\
\hline 3 & 0 & 0.337 & 1.372 & 0.215 & 1.586 & 3.362 & 2.426 & 3.396 & 5.239 & 4.910 \\
\hline 6 & 0 & 0.691 & 1.488 & 0.953 & 2.556 & 4.224 & 4.106 & 4.988 & 6.875 & 7.225 \\
\hline 12 & 0 & 0.867 & 1.355 & 1.465 & 3.132 & 4.437 & 4.920 & 6.051 & 7.828 & 8.676 \\
\hline 1 & 0.1 & 0.068 & 2.752 & 0.006 & 0.541 & 2.803 & 0.527 & 1.394 & 3.382 & 1.979 \\
\hline 3 & 0.1 & 0.922 & 3.918 & 0.151 & 2.396 & 5.045 & 3.262 & 4.176 & 6.411 & 5.912 \\
\hline 6 & 0.1 & 1.834 & 4.152 & 1.514 & 3.850 & 6.347 & 5.703 & 6.160 & 8.461 & 8.758 \\
\hline 12 & 0.1 & 2.420 & 3.908 & 3.685 & 4.790 & 6.767 & 7.188 & 7.592 & 9.783 & 10.695 \\
\hline 1 & 0.2 & 0.122 & 4.763 & 0.011 & 0.764 & 3.888 & 0.687 & 1.708 & 4.113 & 2.377 \\
\hline 3 & 0.2 & 1.513 & 6.510 & 0.100 & 3.217 & 6.757 & 4.128 & 4.968 & 7.604 & 6.936 \\
\hline 6 & 0.2 & 2.983 & 6.844 & 2.112 & 5.153 & 8.489 & 7.330 & 7.342 & 10.063 & 10.311 \\
\hline 12 & 0.2 & 3.979 & 6.478 & 5.936 & 6.456 & 9.109 & 9.477 & 9.141 & 11.748 & 12.729 \\
\hline
\end{tabular}

